\title{
Chaetomium-like fungi causing opportunistic infections in humans: a possible role for extremotolerance
}

\author{
Sarah A. Ahmed ${ }^{1,2,3}$ - Ziauddin Khan ${ }^{4}$ - Xue-wei Wang ${ }^{2,5}$ - Tarek A. A. Moussa ${ }^{6,7}$. \\ Hassan S. Al-Zahrani ${ }^{6}$ - Omar A. Almaghrabi ${ }^{6}$ • Deanna A. Sutton ${ }^{8}$ - S. Ahmad ${ }^{4}$. \\ Johannes Z. Groenewald ${ }^{2}$ - A. Alastruey-Izquierdo ${ }^{9}$ - Anne van Diepeningen ${ }^{2}$. \\ S. B. J. Menken ${ }^{3}$ • M. J. Najafzadeh ${ }^{10}$ • Pedro W. Crous ${ }^{2} \cdot$ Oliver Cornely $^{11}$ • \\ Axel Hamprecht ${ }^{12}$ - Maria J. G. T. Vehreschild ${ }^{11}$ - A. J. Kindo ${ }^{13}$. \\ G. Sybren de Hoog 2,3,6,14,15,16,17
}

Received: 27 March 2015 / Accepted: 11 June 2015 /Published online: 9 July 2015

(C) The Author(s) 2015. This article is published with open access at Springerlink.com

\begin{abstract}
Members of the family Chaetomiaceae are ubiquitous ascosporulating fungi commonly, which reside in soil enriched with manure or cellulosic materials. Their role as human pathogens is largely ignored. However, the ability of some species to grow at high temperature enables them to play an important role as opportunistic pathogens. The family contains several genera and species that have never been reported to cause human infection. Hereby, three new species are
\end{abstract}

Taxonomic novelties: Chaetomium anamorphosum S.A. Ahmed, Z.U. Khan, X. Wang \& de Hoog, Subramaniula asteroides S.A. Ahmed, Z.U. Khan, X. Wang \& de Hoog, Subramaniula obscura S.A. Ahmed, Z.U. Khan, X. Wang \& de Hoog.

Sarah A. Ahmed

s.ahmed@cbs.knaw.nl

1 Faculty of Medical Laboratory Sciences, University of Khartoum, Khartoum, Sudan

2 CBS-KNAW Fungal Biodiversity Centre, P.O. Box 85167, 3508 AD Utrecht, The Netherlands

3 Institute for Biodiversity and Ecosystem Dynamics, University of Amsterdam, Amsterdam, The Netherlands

4 Department of Microbiology, Faculty of Medicine, Kuwait University, Kuwait, Kuwait

5 State Key Laboratory of Mycology, Institute of Microbiology, Chinese Academy of Sciences, Beijing, China

6 Biological Sciences Department, Faculty of Science, King Abdulaziz University, Jeddah, Saudi Arabia

7 Botany and Microbiology Department, Faculty of Science, Cairo University, Giza, Egypt

8 Fungus Testing Laboratory, Department of Pathology, University of Texas Health Science Center at San Antonio, San Antonio, TX, USA described; two belong to the genus Subramaniula and one represents a Chaetomium species. Subramaniula asteroides was isolated from various sources including eye and skin infections as well as from the natural environment, and S. obscura was isolated from a toe infection. Chaetomium anamorphosum was isolated from a kidney transplant patient suffering from fungal peritonitis. All species described were previously misidentified as Papulaspora spp. due to the

9 Mycology Reference Laboratory, National Centre for Microbiology, Carlos III Institute of Health (Servicio de Micología, Centro Nacional de Microbiología, Instituto de Salud Carlos III), Madrid, Spain

10 Department of Parasitology and Mycology, Mashhad University of Medical Sciences, Mashhad, Iran

11 German Centre for Infection Research (DZIF) at Cologne/Bonn, First Department of Internal Medicine, University Hospital of Cologne, Cologne, Germany

12 Institute for Medical Microbiology, Immunology and Hygiene, University of Cologne, Cologne, Germany

13 Department of Microbiology, Sri Ramachandra University, Porur, Chennai, India

14 Peking University Health Science Center, Research Center for Medical Mycology, Beijing, China

15 Sun Yat-Sen Memorial Hospital, Sun Yat-Sen University, Guangzhou, China

16 Shanghai Institute of Medical Mycology, Changzheng Hospital, Second Military Medical University, Shanghai, China

17 Basic Pathology Department, Federal University of Paraná State, Curitiba, Paraná, Brazil 
formation of cellular clumps or bulbil-like structures, which are characteristic of Papulaspora. The isolates failed to form sexual fruit bodies and ascospores remained absent, which is an unusual feature for the generally ascosporulating genera Chaetomium and Subramaniula; minute conidia from phialides were sometimes observed.

Keywords Chaetomium · Desert fungi · Keratitis · Papulaspora $\cdot$ Peritonitis $\cdot$ Sterile fungi $\cdot$ Subramaniula

\section{Introduction}

Melanized fungi are important causes of human infection. About 70 genera representing hundreds of species have been implicated in human disease (de Hoog et al. 2013a; Guppy et al. 1998; Revankar and Sutton 2010; Revankar et al. 2002). Several main ecological groups can be distinguished. Members of Chaetothyriales exhibit pronounced virulence and cause deep and systemic infections in immunocompetent humans, e.g. chromoblastomycosis or brain infection (Badali et al. 2009; Revankar and Sutton 2010). Members of Pleosporales are preponderantly found as degraders of plants debris or as mild opportunistic pathogens; human infections mostly comprise traumatic inoculation of contaminated materials (Revankar and Sutton 2010). Recently, the significance of Sordariales was underlined (Badali et al. 2011; de Hoog et al. 2013b), particularly the Chaetomiaceae whose prevalence has been underestimated because of diagnostic problems.

Phenotypically, the identification of clinical chaetomiumlike fungi has been difficult as a large proportion of them fail to produce typical diagnostic structures in culture (Najafzadeh et al. 2014; Vinod Mootha et al. 2012). Until recently, fungi lacking propagation in the form of conidia were treated in the clinical laboratory as unidentifiable 'mycelia sterilia' (Pounder et al. 2007; Santos et al. 2013; Vinod Mootha et al. 2012). Isolates forming clumps or bulbils were referred to as Papulaspora, whereas filamentous strains from subcutaneous infection were known as Madurella (de Hoog et al. 2013b). With the application of molecular phylogenetic multi-gene DNA sequence analyses, such isolates were found to be of high diversity being distributed to many genera and families of ascomycetes. In addition, species proved to comprise several sibling species that were previously thought to represent a single taxon. For example, Madurella is now known to harbour four species (de Hoog et al. 2012).

Despite recent progress in the taxonomy of sterile fungi, the phylogenetic position of Papulaspora remains uncertain. The occurrence of bulbils or papulospores is non-specific and polyphyletic, as similar compact masses of cells can be produced by a wide range of fungi from different genera and orders (Hotson 1917). The generic type species Papulaspora sepedonioides is a member of the order Melanosporales (Davey et al. 2008), but further papulaspora-like asexual morphs have been assigned to Ceratostoma and Chaetomium in the order Sordariales (Bainier 1907; Hotson 1917; Weresub and LeClair 1971; Zang et al. 2004). Isolates previously misidentified as $P$. sepedonioides are currently listed as non-sporulating Chaetomium species (Vinod Mootha et al. 2012).

Recently, more attention has been paid to the nonsporulating chaetomium-like isolates that cause human infection (Najafzadeh et al. 2014; Vinod Mootha et al. 2012), such as keratitis or subcutaneous infection after trauma. Despite application of molecular phylogenetic methods, researchers were unable to identify these isolates down to species level in the genus Chaetomium because of the present state of morphological confusion and high phylogenetic divergence in the genus. Chaetomium contains more than 300 described species, but modern descriptions of most taxa are lacking, and very few have been circumscribed with DNA data. In the present concept of the genus, species that produce elaborate sporocarps are accepted, and most species lack asexual morphs (von Arx et al. 1986). Species are generally cosmopolitan and reside in soil on cellulose-rich materials or on dung (Bell 2005; Carter and Khan 1982; Doveri 2008; von Arx et al. 1986). A certain prevalence of chaetomium-like species was noted in desert soil subjected to conditions of dryness and extremely variable temperatures (Rodríguez et al. 2004). Members of the madurella-clade phylogenetically located inside the genus Chaetomium are typically confined to areas with arid climates. Madurella species are consistent agents of human subcutaneous mycoses, and the arid areas of northeastern Africa are endemic for human mycetoma (Ahmed et al. 2002). Most human infections by chaetomium-like species concern traumatic inoculations into otherwise healthy humans, and rarely occur as deep infections in severely immunocompromised hosts (Al-Aidaroos et al. 2007; Guppy et al. 1998; Hubka et al. 2011).

In the present paper we describe three novel species from clinical sources that were earlier provisionally identified as Papulaspora or Chaetomium species. One of the species was represented by seven clinical isolates particularly from the Middle East, while an environmental strain was derived from hydrocarbon-polluted desert soil. This raises the question whether in these fungi there is a connection between growth under arid conditions and ability to cause opportunistic infections.

\section{Materials and methods}

\section{Isolates and morphology}

Strains studied were isolated from human patients, or from hydrocarbon-rich desert soil using enrichment under toluene 
atmosphere according to Zhao et al. (2010). Reference strains were obtained from the CBS reference collection (Table 1). Morphology and colony characteristics were examined on Malt Extract Agar (MEA, Oxoid, U.K.) and Oatmeal Agar (OA, home-made at $\mathrm{CBS}$ ) and incubated for 2 weeks at $25{ }^{\circ} \mathrm{C}$ in the dark. Microscopic features were examined on OA, MEA and tap water agar slide cultures. To induce sporulation, isolates were incubated under UV light in a 12-h light/ dark regimen and examined every $7 \mathrm{~d}$ for up to 8 weeks. Mounted slides were examined with a Nikon ECLIPSE $80 \mathrm{i}$ microscope and photographs were captured using a Nikon digital sight DS-5M camera attached to the microscope. Colony growth rates were determined on MEA plates incubated for 1 week at temperatures ranging from 6 to $36^{\circ} \mathrm{C}$ at $3{ }^{\circ} \mathrm{C}$ intervals including 37 and $40{ }^{\circ} \mathrm{C}$.

\section{DNA extraction, amplification and sequencing}

Genomic DNA was extracted using a cetyltrimethyl ammonium bromide (CTAB) method described previously by Möller et al. (1992). Amplification and sequencing were performed for the Internal Transcribed Spacer (ITS) and D1/D2 domains of the 28S rRNA gene, partial translation elongation factor 1- $\alpha$ (TEF1), $\beta$-tubulin (Btub), DNA-dependent RNA polymerase II largest subunit $(R P B 1)$ and second largest subunit $(R P B 2)$. Primers used for amplification and sequencing are according to de Hoog et al. (2013a).

\section{Alignment and phylogenetic analyses}

DNA sequences were assembled and edited using SEQMAN from the Lasergene package (DNASTAR, Madison, WI, U.S.A.). Sequences were deposited in GenBank; accession numbers are listed in Table 1. Two alignments were generated to study the phylogenetic position of the unknown species. Sequences were aligned with the online version of MAFFT v. 7 (http://mafft.cbrc.jp) and manually adjusted using BIOEDIT v. 7.1.3 software (Hall 1999). Each gene was aligned independently and concatenated matrices were prepared using DATACONVERT v. 1.0. The first alignment consisted of ribosomal ITS and LSU sequences of representative species of Chaetomium, Chaetomidium, Thielavia, Papulaspora, Subramaniula, and Madurella. The second alignment consisted of the protein coding loci TEF1, Btub, RPB1 and $R P B 2$ sequences of a selected number of strains. Alignments and trees were deposited in TreeBASE database (TreeBASE ID: 17309).

Phylogenetic analyses using Maximum likelihood were performed in RAxML v. 8.0.24 (Stamatakis 2014). Bayesian analyses with default priori of MRBAYES v. 3.1.2 were conducted using the CIPRES Science Gateway server. Two simultaneous Markov chain Monte Carlo samplings were performed with four chains of which one was cold and three were heated.
The run was conducted for $30,000,000$ generations with sampling every 100 generations and the 'burn in' was set at $25 \%$ of resulting trees. Convergence was evaluated from the two independent runs using AWTY and TRACER v. 1.5 (Nylander et al. 2008; Rambaut and Drummond 2007).

\section{Results}

Phylogenetic analyses of the combined ITS and LSU loci were used to establish the phylogenetic position of the three unknown species. The analyses included 87 sequences with in total 1384 characters, including alignment gaps. In general, the genera of Chaetomiaceae (Chaetomidium, Chaetomium, Subramaniula, Thielavia and the asexual genera Madurella and Papulaspora) could not be resolved (Fig. 1). Chaetomium, Papulaspora and Thielavia were polyphyletic and their taxonomy requires further investigation to define their generic boundaries. Subramaniula and Madurella formed monophyletic (sub)clades within Chaetomium. The ITS and LSU data provided insufficient resolution to ascertain species delimitations. The unidentified isolates clustered in a single, supported clade [Maximum likelihood bootstrap support values (ML-BS) / posterior probabilities (PP) $97 \% / 0.94$ ] in the basal lineages of the Chaetomiaceae. The clade contained Subramaniula thielavioides, which is the type species of Subramaniula and could therefore be regarded as the Subramaniula clade. The clade was phylogenetically distant from Papulaspora equi, represented by the ex-type strain (CBS 573.89), and from P. sepedonioides (strain CBS 265.79), as well as from all described Madurella species. No match was found with any other Chaetomium, Thielavia or related species.

A multi-gene phylogeny with $B t u b, T E F 1, R P B 1$ and $R P B 2$ was used focusing on the Subramaniula clade (Fig. 2). The analysis included 20 taxa with a total of 3571 alignment characters. Three new species were identified, of which two were found to be closely related to Subramaniula thielavioides (ML-BS/PP, $100 \% / 1.0$; Fig. 2) nested within Chaetomium, and were considered to belong to Subramaniula. The proposed species $S$. obscura was a sister to the type species of Subramaniula, S. thielavioides, in a well-supported subclade (ML-BS/ PP, $99 \% / 1.0$ ), whereas $S$. asteroides formed a subclade sister to $S$. thielavioides and S. obscura.

With the four partial protein coding loci, the subcluster of S. asteroides was split into three supported lineages (Fig. 2). The first lineage (ML-BS/PP, $95 \% / 1.0$ ) contained four strains, three of which were of clinical origin and one environmental strain that had been selectively isolated by toluene enrichment from desert soil in Saudi Arabia. The second lineage (ML-BS/ PP, $100 \% / 1.0$ ) contained three isolates from cases of keratitis 


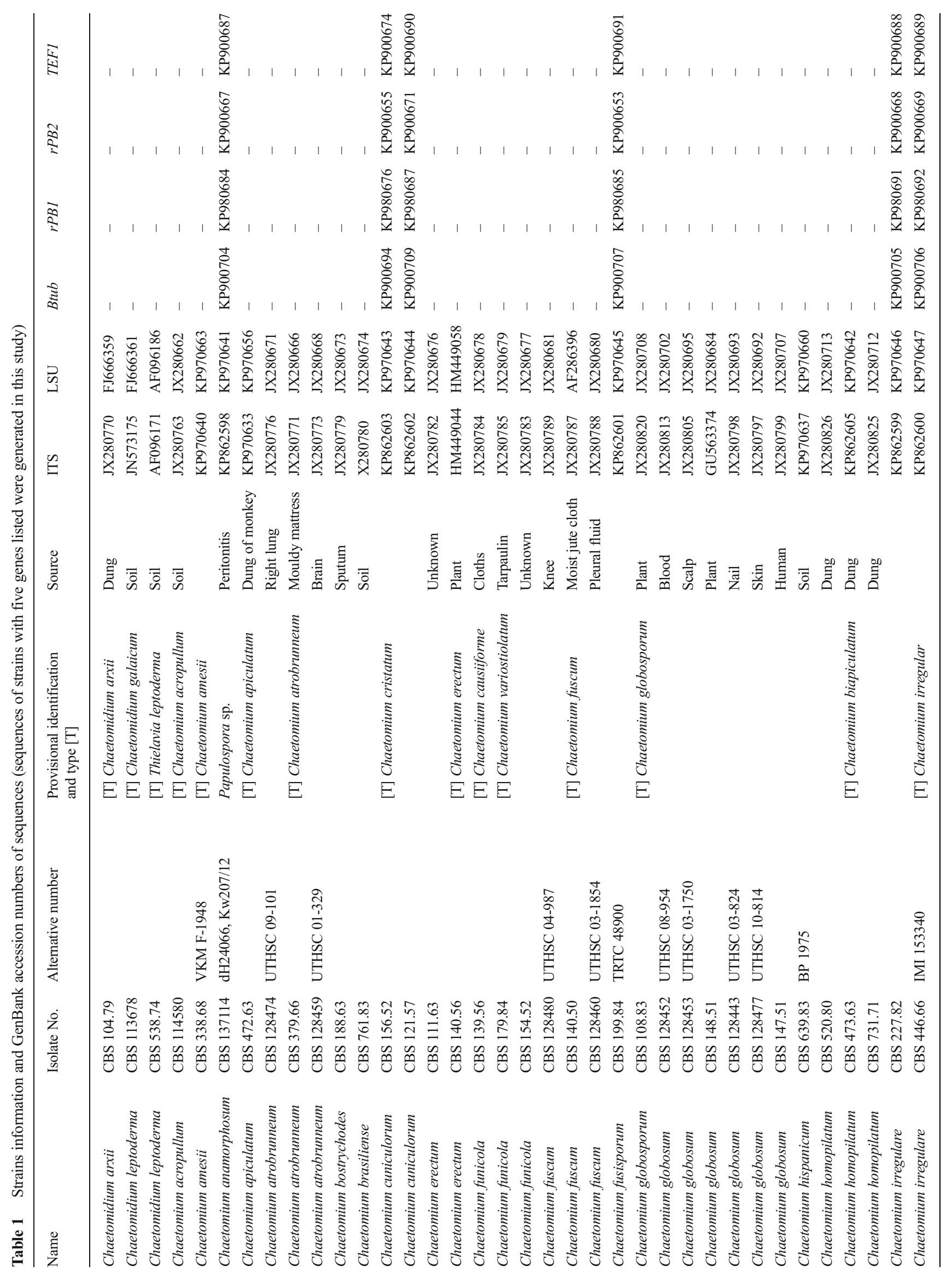




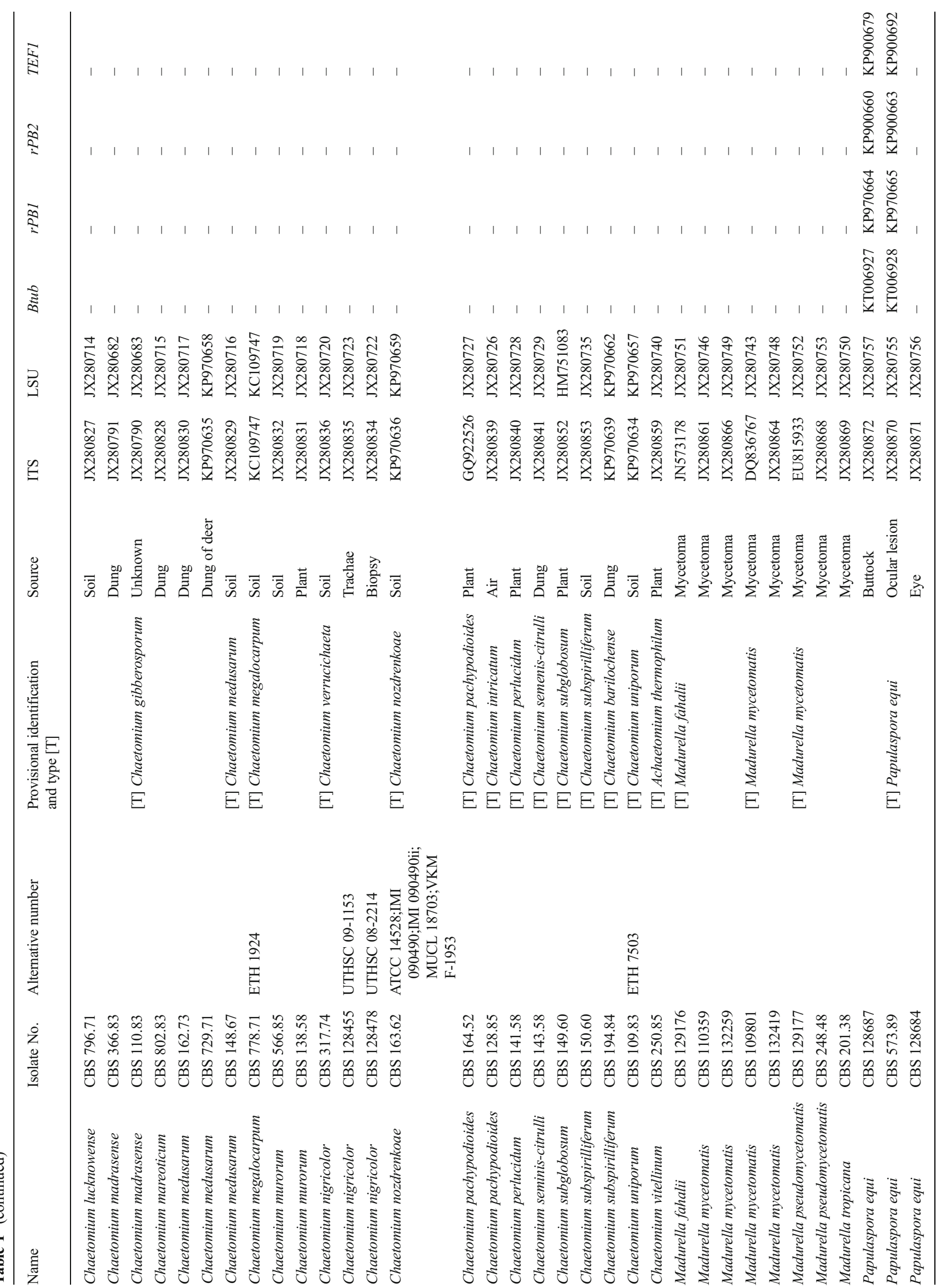




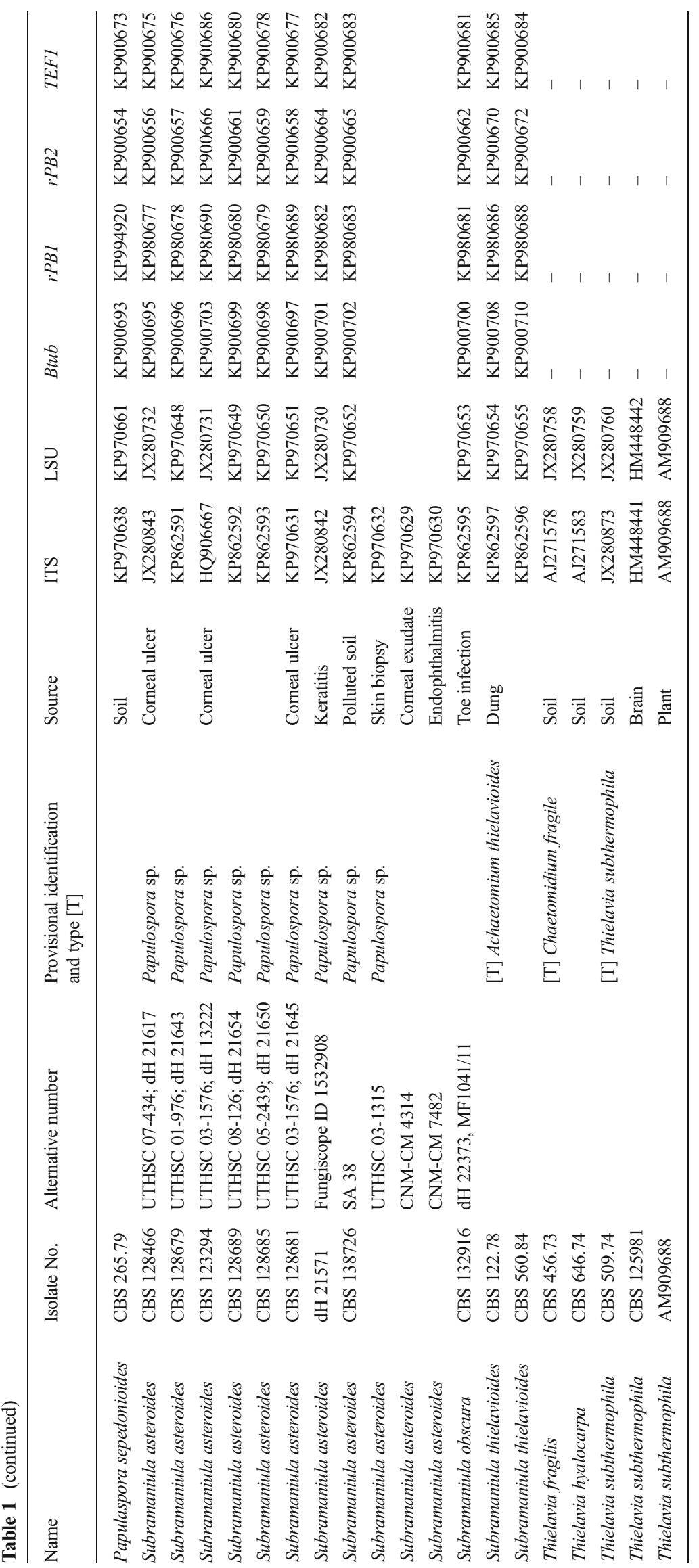


Fig. 1 Phylogram of representative selection of Chaetomiaceae genera obtained by Maximum likelihood and Bayesian analysis of ITS and LSU. Maximum likelihood bootstrap (ML-BS) and Bayesian posterior probability (PP) are indicated at the nodes.

Papulaspora equi was used to root the tree

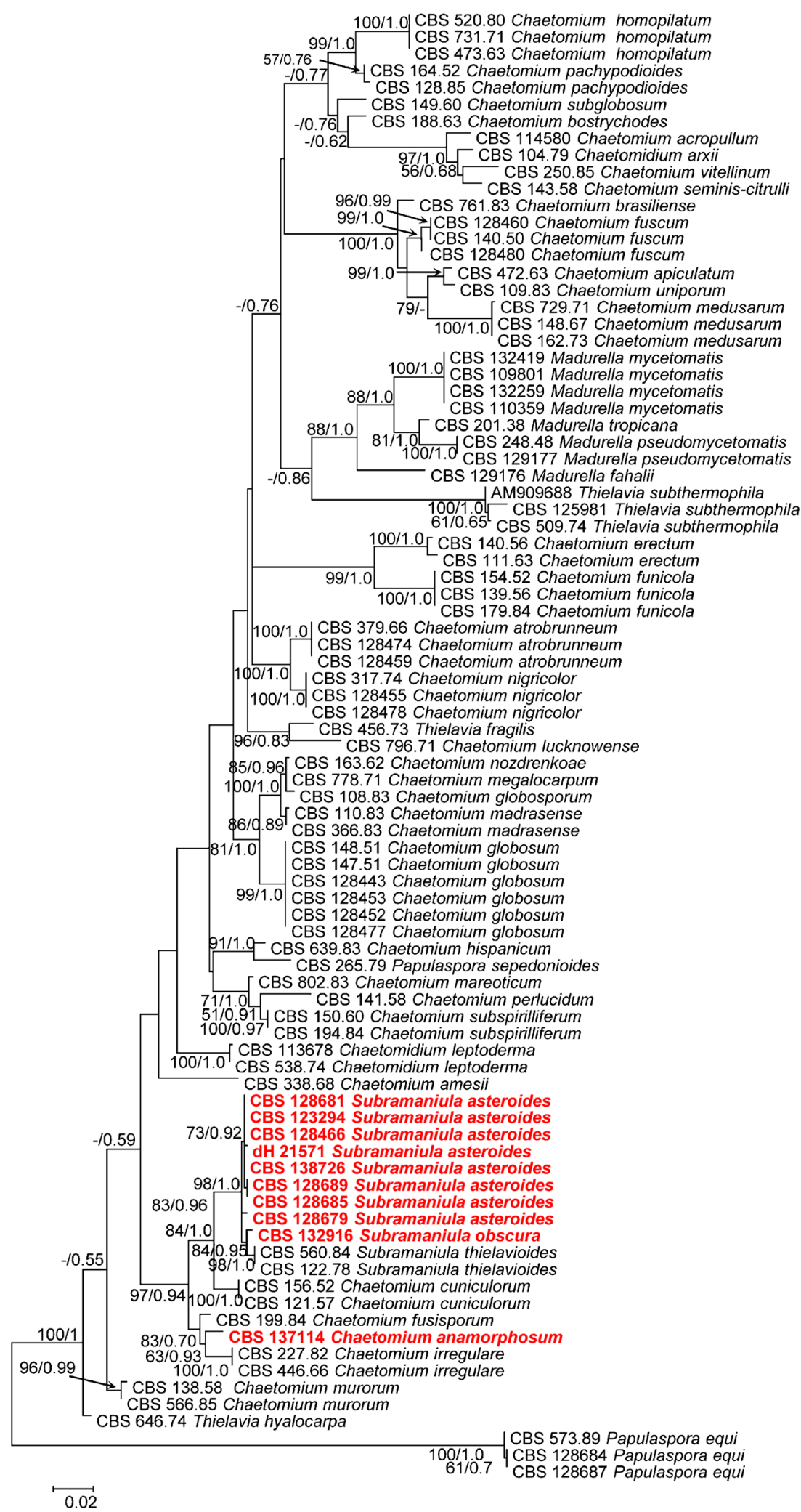

from the U.S.A. The clinical strain from India, $\mathrm{dH} 21571=$ Fungiscope 1532908 deviated slightly from the type.

No match was found for our strains with any existing taxon on NCBI's GenBank nucleotide database. All of our strains are nested within Chaetomium sensu lato (Fig. 1), but distant from the generic type species, C. globosum. The new species proposed in this study cluster sister to strains of Subramaniula thielavioides (the type species of Subramaniula, nestled 
Fig. 2 Phylogeny of combined data $(T E F 1, r P B 1, r P B 2$, and Btub) of Subramanuila clade obtained by Bayesian and Maximum likelihood analysis. Maximum likelihood bootstrap (ML-BS) and Bayesian posterior probability (PP) are indicated at the nodes. Papulaspora equi was used as out group

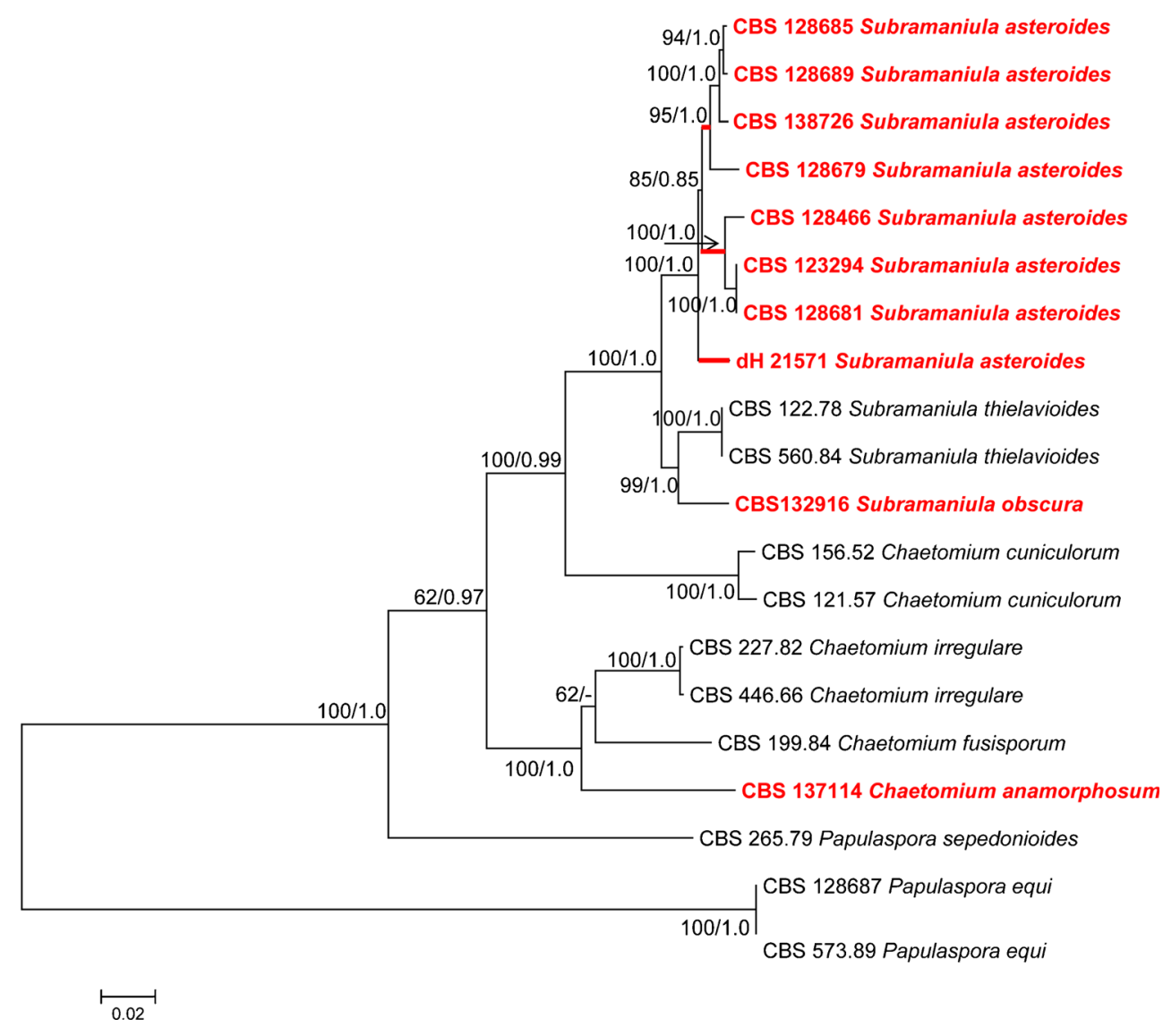

within Chaetomium sensu lato) and are treated in this study as species of Subramaniula, pending a more comprehensive taxonomic study of the Chaetomiaceae. An isolate recovered from a case of peritonitis and originating from Kuwait was found to be closely related to two Chaetomium species, i.e. C. irregulare and C. fusisporum (ML-BS/PP, 100/1.0; Fig. 2); this strain was identified as a novel species, C. anamorphosum (Figs. 1 and 2).

\section{Taxonomy}

Chaetomium anamorphosum S.A. Ahmed, Z. U. Khan, X. Wang \& de Hoog, sp. nov. - Fig. 3, MB 810426

Colonies on MEA velvety, white, becoming yellow to tan with age: reverse buff to yellow. Colonies on OA dark yellowish in the centre becoming faint to colourless toward the margin; mycelium immersed, with a yellow pigment diffusing into the agar. Hyphae hyaline, partially converting to dark brown or black, thick-walled chlamydospore-like structures. Cellular clumps seen on the surface of colonies as small, black, spherical to irregular structures; under the light microscopic they are black, consisting of aggregates of thick-walled cells, 5-10×4-6 $\mu \mathrm{m}$. Phialides hyaline, erect, short cylindrical or broader at the base. Conidia smooth-walled, hyaline, unicellular, 2-3 $\times 1.5-2.0 \mu \mathrm{m}$, obovoidal or ellipsoidal. Minimum growth temperature $6{ }^{\circ} \mathrm{C}$, optimum $33-36{ }^{\circ} \mathrm{C}$, maximum above $40{ }^{\circ} \mathrm{C}$ (Fig. 4).

Holotype: dried culture in CBS Herbarium CBS H-21973; type culture CBS 137114, from case of peritonitis, Kuwait, Z.U. Khan.

\section{Case report of Chaetomium anamorphosum CBS 137114}

A 21-year-old Jordanian patient with a history of end-stage renal disease underwent a kidney transplant in 1995. The transplanted kidney was rejected in 2007. The patient was put on peritoneal dialysis at regular intervals. In February 2011, the patient underwent radical nephrectomy of transplanted and native kidney because of uncontrolled hypertension. In April 2012, the patient presented with complaints of abdominal pain, vomiting and constipation. A CT scan of the abdomen revealed partial small bowel obstruction (ileus). Accompanying symptoms at this time were uncontrolled hypertension and seizures associated with posterior reversible encephalopathy syndrome seen in a CT scan of the brain. He was admitted to the Nephrology Unit of the Mubaral AlKabeer Hospital, Kuwait for further investigation and treatment. A nasogastric tube was placed and about $1500 \mathrm{ml}$ greencoloured fluid was drained. Since the patient developed spiking fever, he was prescribed Tazocin (piperacillin and 

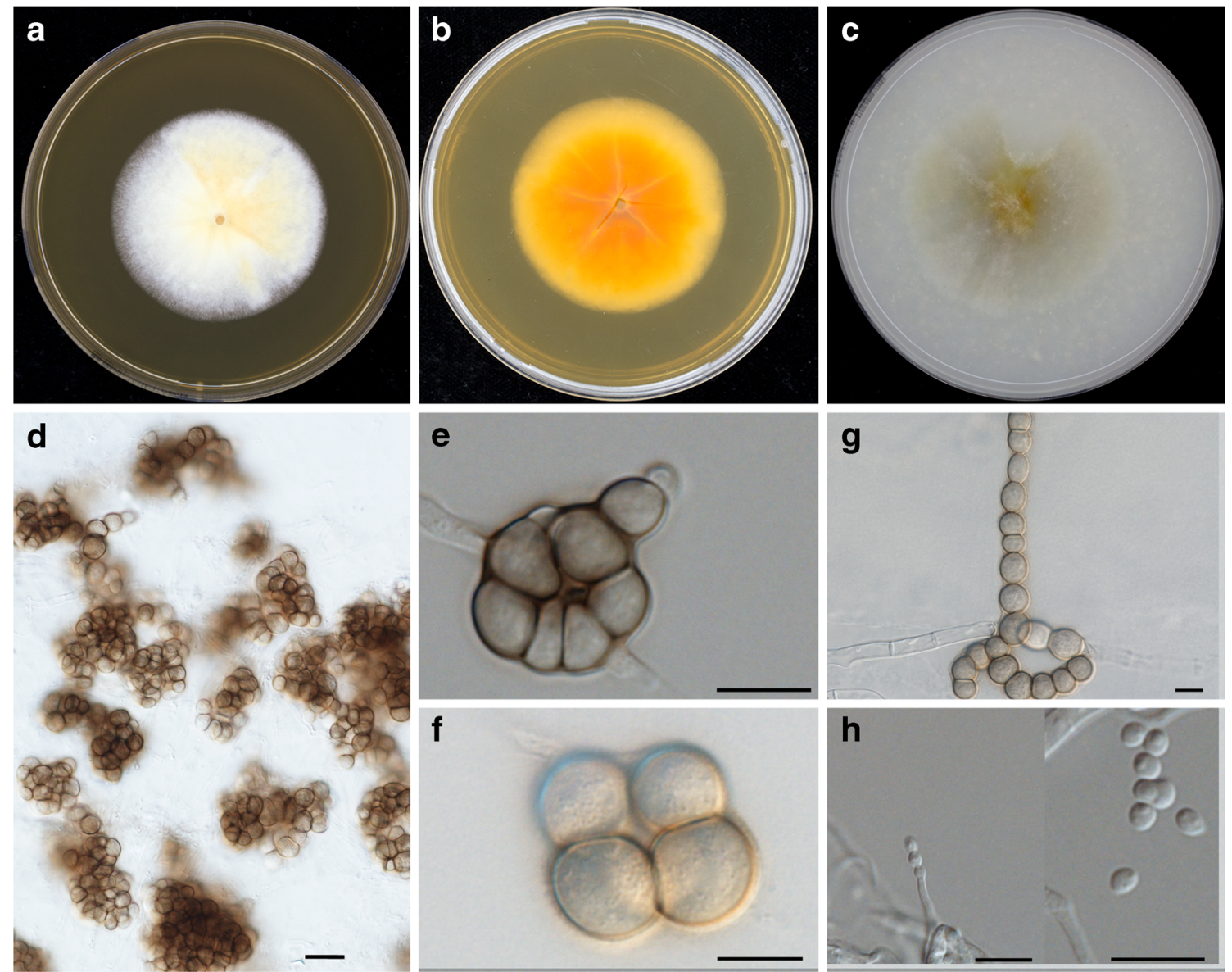

Fig. 3 Chaetomium anamorphosum (CBS 137114). Colonies after 1 week of incubation on: a-b MEA obverse and reverse; $\mathbf{c}$ OA; $\mathbf{d}-\mathbf{f}$ Clumps; $\mathbf{g}$ Thickwalled chlamydospore-like structures; $\mathbf{h}$ Conidiophore and conidia. - Scale bars $=10 \mu \mathrm{m}$

tazobactam) and Flagyl (metronidazole) besides Zantac (ranitidine). Subsequently, the patient developed peritonitis with multiple pockets of fluid collection. Repeated microscopic examination of the centrifuged sediment of the peritoneal fluid, which was turbid, bloody and contained tissue-like flakes, showed septate fungal elements (Fig. 5). The culture of the peritoneal fluid yielded a fungus that grew at $37{ }^{\circ} \mathrm{C}$. The patient was started on voriconazole $400 \mathrm{mg}$, administered twice on day one, followed by $200 \mathrm{mg}$ twice daily for 15 days. He became hypotensive with signs and symptoms of septicemia. The patient was put on hemodialysis, which could not be continued due to severe hypotension. His condition deteriorated rapidly and despite therapy he died of multi-organ failure 1 week later.
Fig. 4 Growth rate of

Chaetomium and Subramaniula species examined after 2 weeks of incubation on $2 \%$ MEA at temperatures ranging from 6 to $40{ }^{\circ} \mathrm{C}$

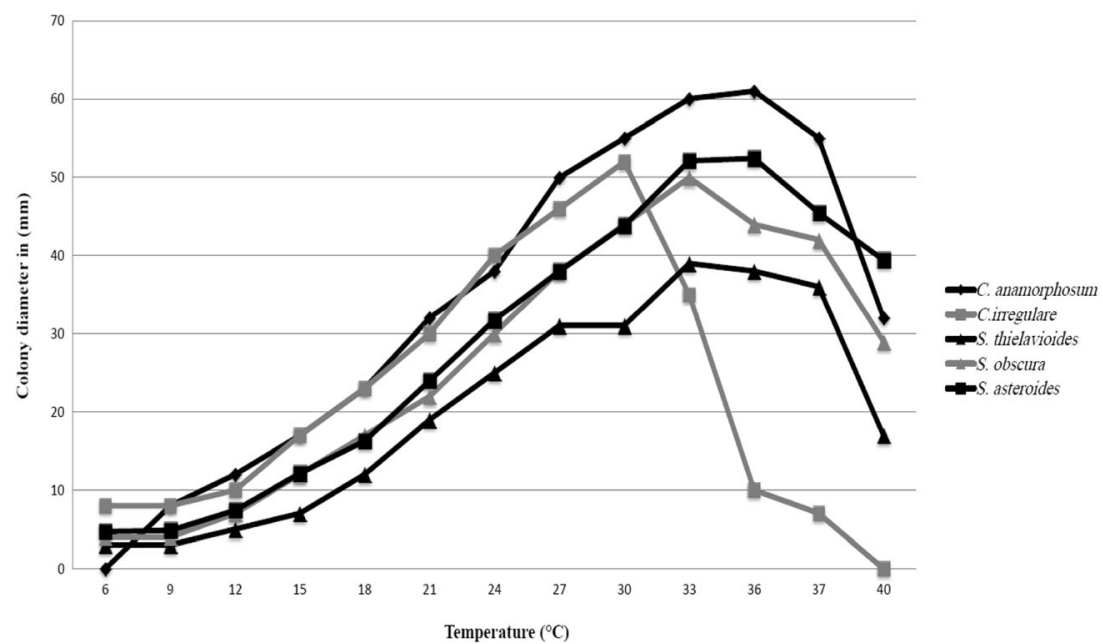




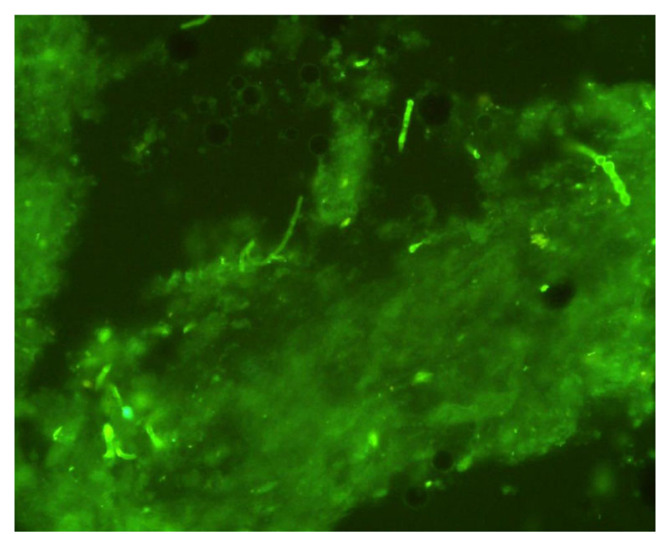

Fig. 5 Direct fluorescence examination of peritoneal dialysis fluid from patient (CBS 137114) showing septate hyphal elements of Chaetomium anamorphosum in Calcofluor mount

Subramaniula asteroides S.A. Ahmed, Z. U. Khan, X. Wang \& de Hoog, sp. nov. - Fig. 6; MB 810427

Colonies on MEA radially folded, yellow green, turning dark greyish green with age; reverse dark grey. Colonies on OA felty, yellowish green or fuscous at the centre becoming faint toward the margin; some isolates showing dark, waxy colonies covered with black bulbils with age. Hyphae broad, septate, hyaline, turning dark brown with age, verruculose; part of the hyphae convert to dark brown or black, thickwalled chlamydospore-like structures. Cellular clumps irregular, black $58-100 \times 44-71 \mu \mathrm{m}$, consisting of aggregates of dark brown, thick-walled cells $7-12 \times 7-9 \mu \mathrm{m}$. Conidiophores phialidic, terminal or intercalary, short, hyaline, obclavate or cylindrical. Conidia hyaline $2-4 \times 1.5-$ $2.0 \mu \mathrm{m}$, unicellular, obovoidal or ellipsoidal. Minimum growth temperature $6{ }^{\circ} \mathrm{C}$, optimum $33-36{ }^{\circ} \mathrm{C}$, maximum above $40{ }^{\circ} \mathrm{C}$ (Fig. 4).

Holotype: dried culture in CBS Herbarium CBS H-21971; type culture CBS 123294, from corneal ulcer, V. Vinod Mootha \& P. Shahinpoor, Chennai, India.

\section{Case reports of Subramaniula asteroides}

(1). A 45-year-old female patient with non-insulin dependent diabetes mellitus presented to the emergency room of the Sri Ramachandra Medical College and Research Institute in Chennai, Tamil Nadu, India, with blepharospasm, photophobia and watering of the right eye. She had suffered from trauma to the right eye by a sharp
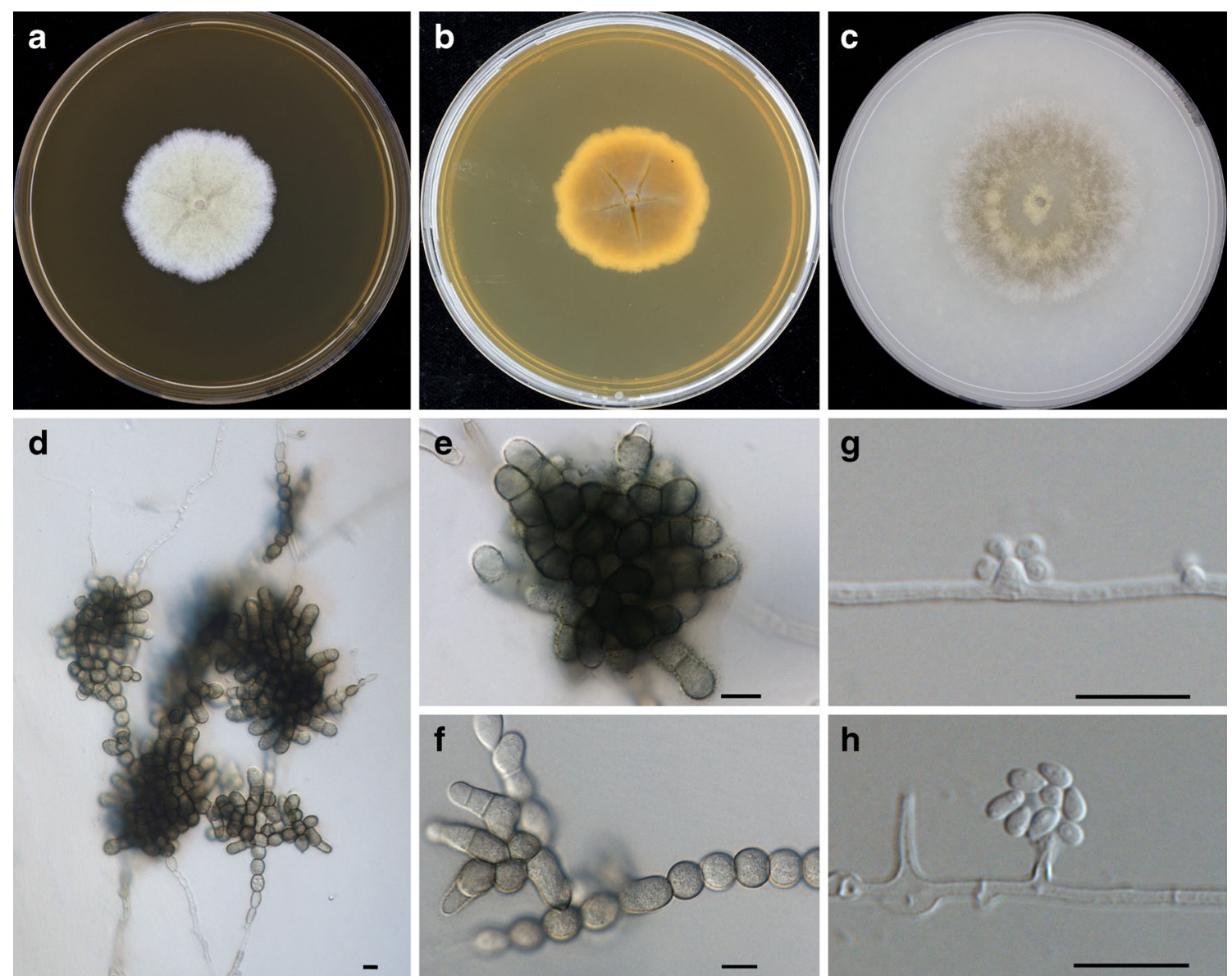

Fig. 6 Subramaniula asteroides (CBS 123294). Colonies after 1 week of incubation on: a-b MEA obverse and reverse; c OA; d-e Clumps; f Thickwalled chlamydospore-like structures; $\mathbf{g}$ Conidiophores and conidia. - Scale bars $=10 \mu \mathrm{m}$ 

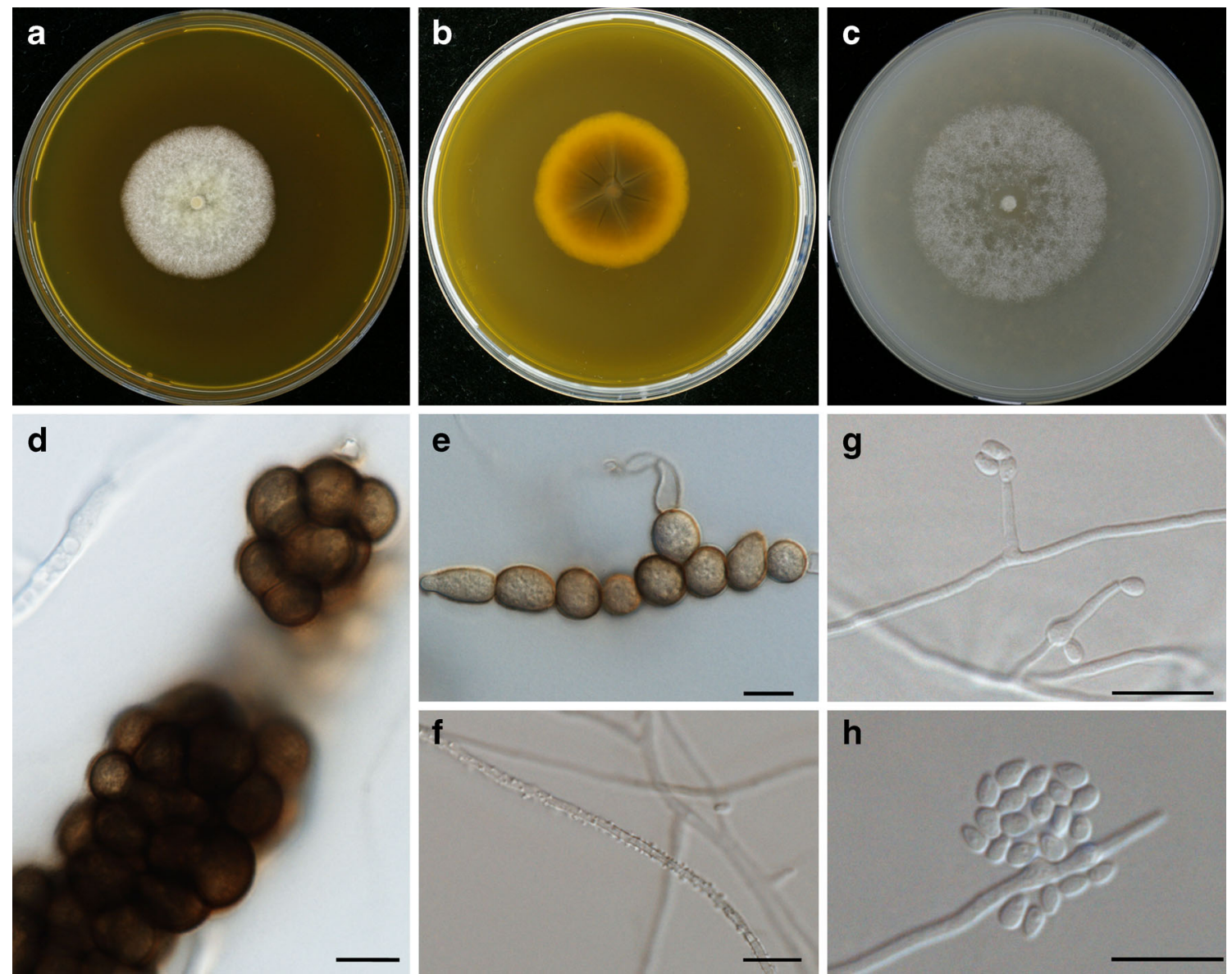

Fig. 7 Subramaniula obscura (CBS 132916). Colonies after 1 week of incubation on: a-b MEA obverse and reverse; $\mathbf{c}$ OA; d Clumps; e Thick-walled chlamydospore-like structures; $\mathbf{f}$ Hyphae with wart like projections; $\mathbf{g}-\mathbf{h}$ Conidiophores and conidia. - Scale bars $=10 \mu \mathrm{m}$

plant leaf during field work 3 days earlier. An eye examination revealed conjunctival congestion as well as a hypopyon. A corneal scraping was carried out. Direct microscopy with $10 \% \mathrm{KOH}$ showed septate hyphae. On Sabouraud's dextrose agar (SDA), white fungal growth was identified. The lactophenol-cotton blue mount showed hyaline hyphae with scytalidium-like arthroconidia of which few were slightly swollen. The patient was treated with amphotericin B deoxycholate $0.15 \%$ eye drops three times daily in combination with fluconazole $200 \mathrm{mg}$ p.o. twice daily for 42 days. Under this treatment, complete recovery of ocular function was obtained.

(2). A 42-year-old male patient without history of diabetes, asthma or use of steroids presented with frequent nasal block for 6 months, particularly on the right side. Symptoms were aggravated due to recurrent upper respiratory bacterial infections with staphyloccocci and Pseudomonas and were temporarily relieved by antibiotics such as augmentin. On local examination patient had a right-sided deviated nasal septum (DNS) with decreased fogging at this side, as demonstrated by cold spatula test. Diagnostic nasal endoscopy showed rightsided DNS with septal spur towards the right side along with blockage of the bilateral ostiomeatal complex. Ear and throat findings were normal. Patient underwent functional endoscopic sinus surgery (FESS) with septoplasty. A tissue sample was sent to the microbiology laboratory for fungal culture. Potassium hydroxide $(\mathrm{KOH})$ mount showed the presence of hyphae and culture on oatmeal agar was positive.

Subramaniula obscura S.A. Ahmed, Z. U. Khan, X. Wang \& de Hoog, sp. nov. - Fig. 7, MB 810428.

Colonies on MEA floccose, greyish green; reverse dark grey. Colonies on OA felty, with fluffy, white to faint grey aerial tufts. Hyphae branched, hyaline, becoming dark brown with age, then thick-walled, verruculose with wart-like projections. Swollen hyphae with brown chlamydospore-like structures $8-13 \times 8-12 \mu \mathrm{m}$ present. Cellular clumps irregular, dark brown, $27-73 \times 20-36 \mu \mathrm{m}$, consisting of spherical to ellipsoidal cells. Phialides erect, hyaline, cylindrical, short or long, terminal or intercalary, often remaining without conidia. Conidia unicellular, hyaline, obovoidal or clavate, $2-3 \times 1.4$ 

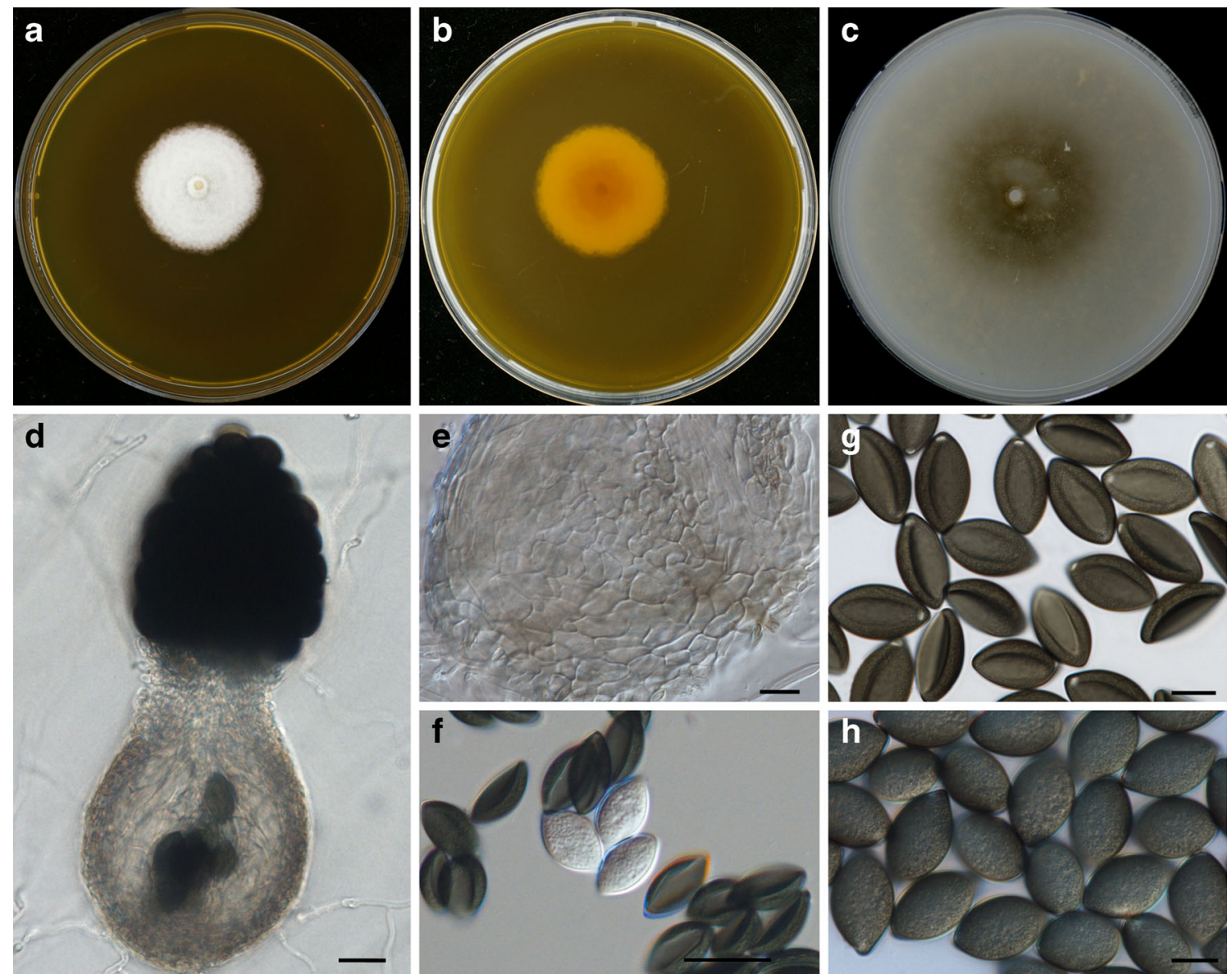

Fig. 8 Subramaniula thielavioides (CBS 122.78). Colonies after 1 week of incubation on: a-b MEA obverse and reverse; c OA; d Ascomata; e Ascomata wall; $\mathbf{f}-\mathbf{h}$ Ascospores. - Scale bars $=10 \mu \mathrm{m}$

$2.0 \mu \mathrm{m}$, forming pseudochains at the tips of conidiophores. Minimum growth temperature $6{ }^{\circ} \mathrm{C}$, optimum $33{ }^{\circ} \mathrm{C}$, maximum above $40{ }^{\circ} \mathrm{C}$ (Fig. 4).

Holotype: dried culture in CBS Herbarium, CBS H-21972; type culture CBS 123916, from male human toe infection, Kuwait, Z.U. Khan.

\section{Discussion}

Identification of filamentous molds in the routine clinical laboratory is still mainly based on microscopic examination of sporulating colonies. Fungi that lack sporocarps or characteristic spores are difficult to identify (Pounder et al. 2007; Santos et al. 2013). In the present study 13 sterile or undiagnostic strains were analyzed. Based on phenotypic features the isolates were identified as Papulaspora species because of the formation of clumps or bulbil-like structures (Vinod Mootha et al. 2012). With attempts to induce sporulation using various substrates and incubation conditions, some strains produced minute conidia on short phialides, but still these conidia were not diagnostically useful and could not be used for species identification.
With molecular and phylogenetic analysis of six loci, the strains were found to belong to three new species which are described in the present study. Two species clustered with the type species of the genus Subramaniula, whereas one was sister to two described Chaetomium species. Both Subramaniula and Chaetomium are members of the family Chaetomiaceae (Sordariales). Members of this family are characterized by the formation of ascospores in deliquescent asci inside more or less extensively ornamented perithecia (Whiteside 1961). Phenotypic identification of species in Chaetomiaceae mostly depends on the shape of ascomatal hairs, ascoma pigmentation and ornamentation, and ascospore shape (Ames 1961; von Arx et al. 1984). The three described species in the present study exhibited unusual morphological features by failure to form sexual reproduction in ascomata. Instead, clusters of thick-walled, dark pigmented cells were present. It is unclear whether these structures represented immature perithecia (Davey et al. 2008). In the framework of singular nomenclature for fungi (Taylor 2011), the species are here nevertheless described in sexual genera on the basis of their phylogenetic position within the Chaetomiaceae.

The two new species clustering with Subramaniula originated from clinical sources, mostly eye or skin infections. The 

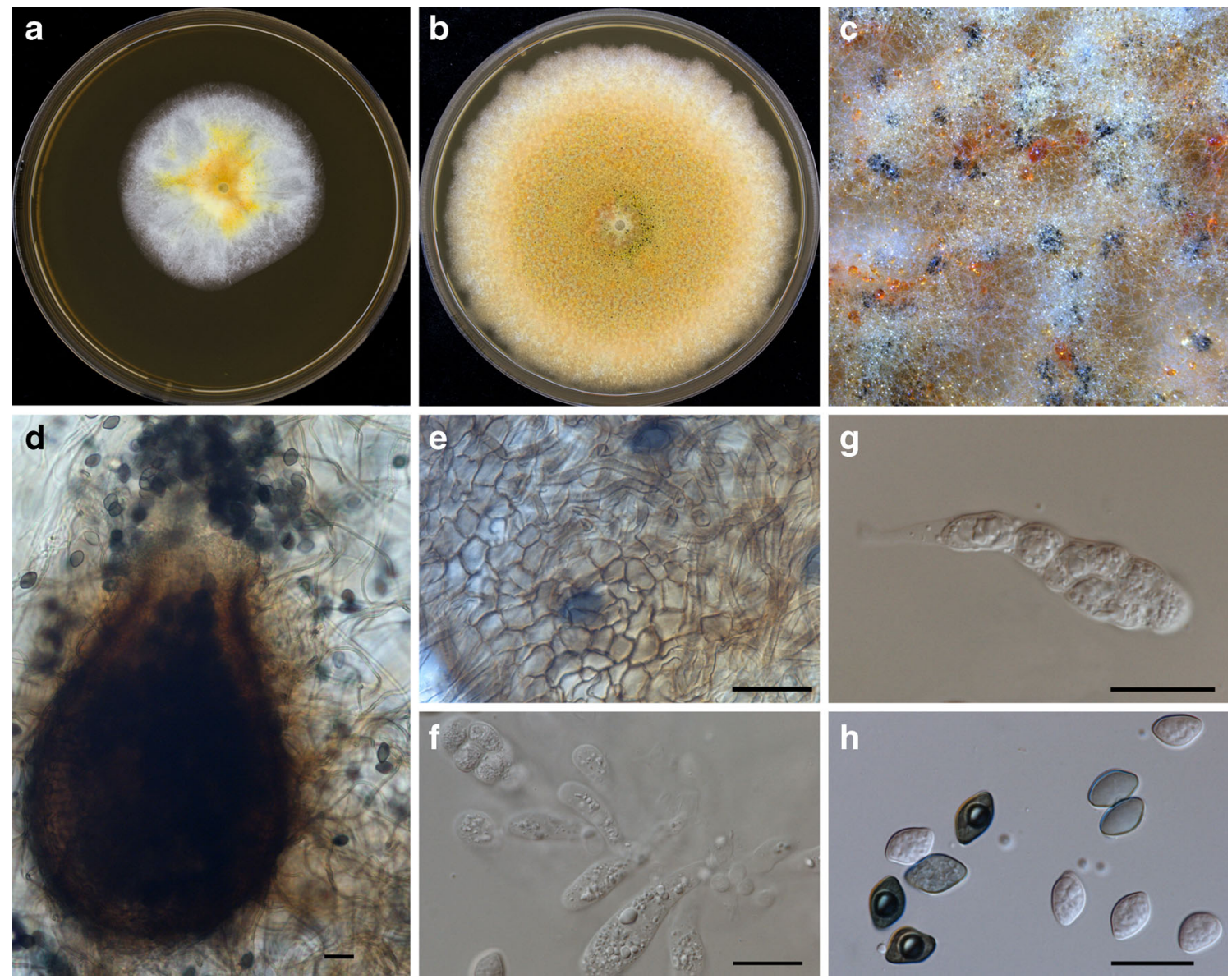

Fig. 9 Chaetomium irregulare (CBS 446.66). Colonies on MEA: a One week b Four weeks of incubation; c Asci on the surface of OA agar; d Ascomata; e Ascomata wall; $\mathbf{f}-\mathbf{g}$ Asci; h. Ascospores. - Scale bars $=10 \mu \mathrm{m}$

coprophilous genus Subramaniula is presently known from two species that were transferred from Achaetomium, i.e. S. irregularis and the generic type species, $S$. thielavioides (Cannon 1986; von Arx 1985; von Arx et al. 1978). The genus Subramaniula is characterized by glabrous pale ascomata with wide apical openings (Fig. 8), without a known asexual morph. Cannon (1986) reported "A few hyphae with chains of considerably swollen cells, producing thin-walled globose or ellipsoidal bodies to $8 \mu \mathrm{m}$ diam, but may well not function as propagule". Some authors suggested that these bodies might be related to the asexual state of this fungus (Pastirčák and Pastirčáková 2009). Upon the examination of the ex-type strain of $S$. thielavioides (CBS 122.78), we detected chains of swollen cells, but no thin-walled bodies were seen. Compared with our two novel Subramaniula species in which conidia were observed, these structures are unlikely to be the conidia, but may act as survival propagules. Subramaniula irregularis (syn. Achaetomiella irregulare) is described as morphologically similar to $S$. thielavioides with some differences in shape and size of ascospores and pigmentation of ascomata but no living culture is available for this species (Cannon 1986).
Subramaniula thielavioides has been isolated from a dung sample from India, whereas $S$. irregularis is only known from soil in South Africa (Cannon 1986; von Arx et al. 1978). Thus far no human infection has been sttributed to Subramaniula. However, Cannon (1986) reported the isolation of S. thielavioides from a human nail and interestingly he reported "Medical mycologists should be aware of its existence". With the two newly described clinical species, the genus Subramaniula is confirmed to have a potential as an opportunistic genus eventually causing skin, eye, and nail infections. An unidentified strain from a corneal ulcer published by Vinod Mootha et al. (2012), CBS 123294, is described in the present study as a novel species Subramaniula asteroides. The strain was recovered from a patient wearing a contact lens and who was injured with a wire while working in a horse stable. Strain dH 21571 concerned a keratitis emerging after eye trauma with a sharp plant leaf on an agricultural field in tropical India. Two further strains from the U.S.A. were also derived from corneal ulcers. Two more strains from eye infections in Spain matched with S. asteroides; CNM-CM 4314 was isolated from corneal exudate of a 27 -year-old male patient, CNM-CM 7482 originated from an endophthalmitis of a 
47-year-old immunosuppressed patient; hyphyae were observed in the vitreous humour and the patient lost the eye. Subramaniula asteroides thus seems to have a strong association with traumatic eye infections. During the course of this study another sequence derived from an isolate (UTHSC 031315) from skin of a patient from Saudi Arabia was also identified as $S$. asteroides. The only environmental isolate of S. asteroides also originated from Saudi Arabia, namely from sandy desert soil under hydrocarbon impact. It was recovered by toluene enrichment (Zhao et al. 2010), a method designed for the isolation of fungi growing under toxic conditions.

Subramaniula obscura is described in the present paper for a single strain from a 53-year-old Kuwaiti male present with a toe infection. The strain was initially identified as Chaetomium cuniculorum. We found that strains of C. cuniculorum are phylogenetically related to S. obscura, as both were found in a single, supported clade based on both ribosomal gene analysis (ML-BS/PP, $84 \% / 1.0$ ) and multilocus analysis of protein coding genes (ML-BS/PP, $100 \% / 0.99$ ) (Fig. 2). With ITS sequencing and using a limited number of taxa, separation of the two species might indeed be difficult. The ITS sequence of the ex-type strain of S. obscura, CBS 132916, showed $97 \%$ similarity to C. cuniculorum isolates. The latter species is thought to occur mostly on dung and about half of the C. cuniculorum strains maintained in the CBS culture collection originated from dung of herbivorous animals. ITS similarity of $S$. obscura to $S$. thielavioides is $99.4 \%$, but protein coding genes are consistently different and therefore description of the novel species in Subramaniula is warranted.

The macro- and micro-morphology of Subramaniula obscura is similar to that of $S$. asteroides except in that the cells forming the clumps of $S$. obscura are more brownish and rounded, and hyphae are thicker and with wart-like protrusions. The minimum growth temperature for both species and for S. thielavioides is $6{ }^{\circ} \mathrm{C}$, and they all grew very well above $40{ }^{\circ} \mathrm{C}$ indicating thermotolerance of Subramaniula species. Optimum growth was between 33 and $36{ }^{\circ} \mathrm{C}$, and consistent survival at $37{ }^{\circ} \mathrm{C}$ denotes the ability to grow at human body temperature as virulence factor (Revankar and Sutton 2010).

Identification of Chaetomium anamorphosum strain CBS 137114 as a novel species, recovered from human peritonitis, was initially done by morphological comparison with described Papulaspora species. This strain showed some similarity to Papulaspora nishigaharanas by formation of aggregates of brown-coloured, thick-walled cells, as well as by production of phialoconidia (Watanabe 1991). Comparison of sequences of ITS and of the D1/D2 region of 28S rRNA gene in GenBank did not reveal homology with any known sequenced species. With the molecular phylogenetic approach we were able to resolve the taxonomy of this isolate as a close relative of the ascosporulating species Chaetomium irregulare and C. fusisporum (Figs. 1, 2, and 9). The newly identified species differed from C. irregulare and C. fusisporum, not only in morphology but also in the growth temperature. The optimum growth temperature of $C$. anamorphosum was 33$36{ }^{\circ} \mathrm{C}$ and growth was still observed at $40{ }^{\circ} \mathrm{C}$, whereas for C. irregulare the optimum was $30^{\circ} \mathrm{C}$ and it was unable to grow or grew only poorly at $40{ }^{\circ} \mathrm{C}$. Chaetomium irregulare was transferred to the genus Achaetomium by Rodríguez et al. (2004). However, when compared with the generic type Achaetomium globosum, it was found to be distant and the name Achaetomium irregulare is redundant. The taxonomy and nomenclature of the clade containing the new species and other Chaetomium spp. as paraphyletic to Subramaniula are unclear because of the relatively large distance to the generic type species, $C$. globosum. In addition, the morphology of ascomata, ascomatal hairs if present, and ascospores of the species of this clade differs from that of C. globosum. The genera Chaetomium, Achaetomium, Subramaniula, and Achaetomiella were morphologically similar with only few differences in the presence and absence of ascomatal hairs, ascoma wall, ascospore color, and in growth rate (Cannon 1986). These characters have become largely irrelevant due to the availability of molecular data. Pending a taxonomic revision of Chaetomium, we maintain the current classification of the cluster within this genus, acknowledging Subramaniula as a genus nested within Chaetomium.

Despite the large number of described species in Chaetomium and relatives, little is known about their molecular taxonomy and only few species have been sequenced and are available in public databases (Wang et al. 2014). Most of the previous studies were restricted to rare species, and the phylogeny of more ubiquitous Chaetomium species remains unresolved (Asgari and Zare 2011; Lee and Hanlin 1999; Wang et al. 2014). Recently, three new species in the C. indicum group were described based on molecular analysis of four genes (Wang et al. 2014). Another molecular study of Chaetomium was that of Asgari and Zare (2011) in Iran using phylogenetic analysis of three genes resulting in the delimitation of six new species. Two of these showed asexual morphs similar to those formed by Chaetomium anamorphosum and by both Subramaniula species described in the present paper. Chaetomium rectangulare Asgari and Zare (2011) belongs to the $C$. globosum group characterized by asexual morphs with phialides and conidia closely similar to those of our new species. This might indicate that the new species somehow lost their ability to form sexual fruiting and replaced this by an asexual type of sporulation. Sequencing of the MAT locus regulating sexual reproduction might indicate whether the sexual morphs in these species are absent or suppressed.

Animal dung, decaying vegetable matter and soil are known natural habitats for Chaetomium, Subramaniula and Papulaspora species. De Hoog et al. (2013b) noticed that the genus Madurella is nested within Chaetomium and that many species in the Chaetomiaceae combine dung- 
association with arid climatic conditions. Adding the previously unidentified non-ascosporulating species to Chaetomium, the role of this genus in human and animal disease has increased significantly. Chaetomium infections and infections by species clustering in the Chaetomium phylogenetic tree, such as Chaetomidium and Thielavia, have been reported from skin, hair, and nails (Hubka et al. 2011; Kaliamurthy et al. 2011; Najafzadeh et al. 2014; Vinod Mootha et al. 2012). Moreover, several species of the Chaetomiaceae have been reported to cause serious opportunistic infections in immunocompromised patients (AlAidaroos et al. 2007; Guppy et al. 1998; Hoppin et al. 1983). Several cases of peritonitis similar to ours in C. anamorphosum have been reported in immunocompromised individuals who had acquired the infection during peritoneal dialysis. Febré et al. (1999) isolated C. globosum from bottles of dialysis fluid; peritonitis was reported by Baer et al. (2013). An interesting case published by Issa et al. (2013) concerned a peritonitis in an immunocompetent female from Damam, Saudi Arabia, caused by an unknown Chaetomium species. Apparently also immunocompetent individuals are at risk of deep infections by Chaetomium or related fungi. As an example, a case of fatal cerebral phaeohyphomycosis caused by Thielavia subthermophila was reported in immunocompetent individuals (Badali et al. 2011). Chaetomium atrobrunneum, C. perlucidum, and C. strumarium were now regarded as neurotropic species causing serious and lifethreatening infections (Abbott et al. 1995; Barron et al. 2003; Guppy et al. 1998). It seems that members of Chaetomiaceae indeed have an underestimated clinical potential, and re-evaluation of the role of the genus in human pathology is urgently required. The natural habitat of many species in arid climates and their survival of high temperatures probably enhance their survival in mammalian tissue. The ability of $C$. anamorphosum to grow optimally at $36{ }^{\circ} \mathrm{C}$, in contrast to $C$. irregulare, is a good example. The species are able to break the thermal exclusionary zone of the human body and if there is immunosuppression or other immunological problem fungi can emerge as potential human pathogens (Casadevall 2012).

Due to identification difficulties on the basis of phenotypic criteria, some older cases of Chaetomium or Subramaniula species might have erroneously been disregarded or reported as cases of Madurella or Papulaspora infection (Mohd-Tahir et al. 2012). Our studies clearly show that traumatic and opportunistic infections by chaetomium-like species often yield non- or poorlysporulating strains in culture (Najafzadeh et al. 2014; Vinod Mootha et al. 2012). Description of these strains as separate taxonomic entities in the genus is significant from both clinical and epidemiological points of view. Moreover, antifungal susceptibility studies are scant, and treatment protocols are urgently needed.
Acknowledgments This project was funded by the Deanship of Scientific Research (DSR) at King Abdulaziz University, Jeddah, under grant No. 1-965/1434 HiCi. The authors, therefore, acknowledge with thanks DSR technical and financial support. We are indebted to the Fungiscope team at Cologne, Germany for stimulating the collection and preservation of uncommon clinical cases. We thank Leena Joseph for technical help and Dr. R. K. Gupta for providing clinical details.

Open Access This article is distributed under the terms of the Creative Commons Attribution 4.0 International License (http:// creativecommons.org/licenses/by/4.0/), which permits unrestricted use, distribution, and reproduction in any medium, provided you give appropriate credit to the original author(s) and the source, provide a link to the Creative Commons license, and indicate if changes were made.

\section{References}

Abbott SP, Sigler L, McAleer R, McGough DA, Rinaldi MG, Mizell G (1995) Fatal cerebral mycoses caused by the ascomycete Chaetomium strumarium. J Clin Microbiol 33:2692-2698

Ahmed A, Adelmann D, Fahal A, Verbrugh H, van Belkum A, de Hoog GS (2002) Environmental occurrence of Madurella mycetomatis, the major agent of human eumycetoma in Sudan. J Clin Microbiol 40:1031-1036

Al-Aidaroos A, Bin-Hussain I, El Solh H, Kofide A, Thawadi S, Belgaumi A, Al Ahmari A (2007) Invasive Chaetomium infection in two immunocompromised pediatric patients. Pediatr Infect Dis J $26: 456-458$

Ames LM (1961) A monograph of the Chaetomiaceae. US Army Res Dev Ser 2. 125 pp

Asgari B, Zare R (2011) The genus Chaetomium in Iran, a phylogenetic study including six new species. Mycologia 103:863-882

Badali H, Carvalho VO, Vicente V, Attili-Angelis D, Kwiatkowski IB, Gerrits van den Ende AHG, de Hoog GS (2009) Cladophialophora saturnica sp. nov., a new opportunistic species of Chaetothyriales revealed using molecular data. Med Mycol 47:51-62. doi:10.1080/ 13693780802291452

Badali H, Chander J, Gupta A, Rani H, Punia RS, de Hoog GS, Meis JF (2011) Fatal cerebral phaeohyphomycosis in an immunocompetent individual due to Thielavia subthermophila. J Clin Microbiol 49: 2336-2341. doi:10.1128/JCM.02648-10

Baer RA, Killen JP, Cho Y, Mantha M (2013) Non-candidal fungal peritonitis in Far North Queensland: a case series. Perit Dial Int 33:559 564. doi:10.3747/pdi.2012.00024

Bainier G (1907) Evolution du Papulaspora aspergilliformis et étude de deux Ascodesmis nouveaux. Bull Trimest Soc Mycol Fr 23:132

Barron MA, Sutton DA, Veve R, Guarro J, Rinaldi M, Thompson E, Cagnoni PJ, Moultney K, Madinger NE (2003) Invasive mycotic infections caused by Chaetomium perlucidum, a new agent of cerebral phaeohyphomycosis. J Clin Microbiol 41:5302-5307

Bell A (2005) An illustrated guide to the coprophilous Ascomycetes of Australia. CBS Fungal Biodivers Ser 3:1-172

Cannon PF (1986) A revision of Achaetomium, Achaetomiella and Subramaniula, and some similar species of Chaetomium. Trans $\mathrm{Br}$ Mycol Soc 87:45-76

Carter A, Khan RS (1982) New and interesting Chaetomium species from East Africa. Can J Bot 60:1253-1262

Casadevall A (2012) Fungi and the rise of mammals. PLoS Pathog 8, e1002808. doi:10.1371/journal.ppat. 1002808

Davey ML, Tsuneda A, Currah RS (2008) Evidence that the gemmae of Papulaspora sepedonioides are neotenous perithecia in the Melanosporales. Mycologia 100:626-635 
de Hoog GS, van Diepeningen AD, el Mahgoub S, van de Sande WW (2012) New species of Madurella, causative agents of black-grain mycetoma. J Clin Microbiol 50:988-994

de Hoog G, Guarro J, Gené J, Figueras MJ (2013a) Atlas of Clinical Fungi, 3rd edn. (e-version). Centraalbureau voor Schimmelcultures, Utrecht, The Netherlands / Universitat Rovira $i$ Virgili, Reus, Spain

de Hoog GS, Ahmed SA, Najafzadeh MJ, Sutton DA, Saradeghi Keisari M, Fahal AH, Eberhart U, Verkley GJ, Xin L, Stielow B, van de Sande WWJ (2013b) Phylogenetic findings suggest possible new habitat and routes of infection of human eumyctoma. PLoS Negl Trop Dis 7, e2229. doi:10.1371/journal.pntd.0002229

Doveri F (2008) An update on the genus Chaetomium with descriptions of some coprophilous species, new to Italy. Pagine Micol 29:1-60

Febré N, Silva V, Medeiros EA, Godoy P, Reyes E, Halker E, Fischman O (1999) Contamination of peritoneal dialysis fluid by filamentous fungi. Rev Iberoam Micol 16:238-239

Guppy KH, Thomas C, Thomas K, Anderson D (1998) Cerebral fungal infections in the immunocompromised host: a literature review and a new pathogen Chaetomium atrobrunneum: case report. Neurosurgery 43:1463-1469

Hall TA (1999) BioEdit: a user-friendly biological sequence alignment editor and analysis program for Windows 95/98/NT. Nucleic Acids Symp Ser 41:95-98

Hoppin EC, McCoy EL, Rinaldi MG (1983) Opportunistic mycotic infection caused by Chaetomium in a patient with acute leukemia. Cancer 52:555-556

Hotson JW (1917) Notes on bulbiferous fungi with a key to described species. Bot Gaz 64:265-284

Hubka V, Mencl K, Skorepova M, Lyskova P, Zalabska E (2011) Phaeohyphomycosis and onychomycosis due to Chaetomium spp., including the first report of Chaetomium brasiliense infection. Med Mycol 49:724-733. doi:10.3109/13693786.2011.572299

Issa H, Alghamdi A, Aljishi YA, Shorman M, Al-Salem A (2013) Chaetomium peritonitis in an immunocompetent patient simulating tuberculous peritonitis: a case report and review of the literature. Microbiol Res Int 1:1-5

Kaliamurthy J, Kalavathy CM, Nelson JCA, Thomas PA (2011) Keratitis due to Chaetomium sp. Case Rep Ophthalmol Med. 696145. doi: 10. $1155 / 2011 / 696145$

Lee S, Hanlin RT (1999) Phylogenetic relationships of Chaetomium and similar genera based on ribosomal DNA sequences. Mycologia 91: 434- 442

Mohd-Tahir F, Norhayati A, Siti-Raihan I, Ibrahim M (2012) A 5-year retrospective review of fungal keratitis at Hospital Universiti Sains Malaysia. Interdiscip Perspect Infect Dis 2012:851563. doi:10.1155/ 2012/851563

Möller EM, Bahnweg G, Sandermann H, Geiger HH (1992) A simple and efficient protocol for isolation of high molecular weight DNA from filamentous fungi, fruit bodies, and infected plant tissues. Nucleic Acids Res 20:6115-6116

Najafzadeh MJ, Fata A, Naseri A, Keisari MS, Farahyar S, Ganjbakhsh M, Ziaee M, Dolatabadi S, de Hoog GS (2014) Implantation phaeohyphomycosis caused by a non-sporulating Chaetomium species. J Mycol Med 24:161-165. doi:10.1016/j.mycmed.2013.09. 007
Nylander JA, Wilgenbusch JC, Warren DL, Swofford DL (2008) AWTY: a system for graphical exploration of MCMC convergence in Bayesian phylogenetic inference. Bioinformatics 24:581-583

Pastirčák M, Pastirčáková K (2009) European record of Subramaniula thielavioides on opium poppy. Acta Mycol 44:7-9

Pounder J, Simmon KE, Barton CA, Hohmann SL, Brandt ME, Petti CA (2007) Discovering potential pathogens among fungi identified as non-sporulating molds. J Clin Microbiol 45:568-571

Rambaut A, Drummond AJ (2007) Tracer v. 1.4. Available from http:// beast.bio.ed.ac.uk/tracer. Accessed Sept 292014

Revankar SG, Sutton DA (2010) Melanized fungi in human disease. Clin Microbiol Rev 23:884-928. doi:10.1128/CMR.00019-10

Revankar SG, Patterson JE, Sutton DA, Pullen R, Rinaldi MG (2002) Disseminated phaeohyphomycosis: review of an emerging mycosis. Clin Infect Dis 34:467-476

Rodríguez K, Stchigel AM, Cano JF, Guarro J (2004) A new species of Achaetomium from Indian soil. Stud Mycol 50:77-82

Santos DW, Padovan AC, Melo AS, Gonçalves SS, Azevedo VR, Ogawa MM, Freitas TV, Colombo AL (2013) Molecular identification of melanised non-sporulating moulds: a useful tool for studying the epidemiology of phaeohyphomycosis. Mycopathologia 175:445454. doi:10.1007/s11046-012-9608-x

Stamatakis A (2014) RAxML Version 8: a tool for phylogenetic analysis and post-analysis of large phylogenies. Bioinformatics 30:13121313. doi:10.1093/bioinformatics/btu033

Taylor JW (2011) One Fungus = One Name: DNA and fungal nomenclature twenty years after PCR. IMA Fungus 2:113-120. doi:10. 5598/imafungus.2011.02.02.01

Vinod Mootha V, Shahinpoor P, Sutton DA, Xin L, Najafzadeh MJ, de Hoog GS (2012) Identification problems with sterile fungi, illustrated by a keratitis due to a non-sporulating chaetomium-like species. Med Mycol 50:361-367. doi:10.3109/13693786.2011.611179

von Arx JA (1985) On Achaetomium and a new genus Subramaniula (Ascomycota). Proc Indiana Acad Sci 94:341-345

von Arx JA, Mukerji KG, Singh N (1978) A new coprophilous ascomycete from India. Persoonia 10:144-146

von Arx JA, Dreyfuss M, Müller E (1984) A revaluation of Chaetomium and Chaetomiaceae. Persoonia 12:169-179

von Arx JA, Guarro J, Figueras MJ (1986) The ascomycete genus Chaetomium. Beih Nova Hedwig 84:1-162

Wang XW, Wang XL, Liu FJ, Zhao XM, Li J, Cai L (2014) Phylogenetic assessment of Chaetomium indicum and allied species, with the introduction of three new species and epitypification of $C$. funicola and C. indicum. Mycol Prog 13:719-732. doi:10.1007/s11557-0130955-x

Watanabe T (1991) New species of Oedocephalum and Papulaspora from Japanese soils. Mycologia 83:524-529

Weresub LK, LeClair PM (1971) On Papulaspora and bulbilliferous basidiomycetes Burgoa and Minimedusa. Can J Bot 49:2203-2213

Whiteside WC (1961) Morphological studies in the Chaetomiaceae. I. Chaetomium. Mycologia 53:512-523

Zang M, Wang R-L, Hu H (2004) Bulbils exist in root of Cypripedium flavum. Acta Bot Yunnan 26:495-496

Zhao J, Zeng J, de Hoog GS, Attili-Angelis D, Prenafeta-Boldú FX (2010) Isolation and identification of black yeasts by enrichment on atmospheres of monoaromatic hydrocarbons. Microb Ecol 60: 149-156. doi:10.1007/s00248-010-9651-4 